\title{
Malaria: the Prospects and Challenges of Eradication A Review
}

\author{
By \\ *Daboer, J.C. **Bot T.C.
}

*Department of Community Medicine, University of Jos/Jos University Teaching Hospital, Jos

*Department of Community Medicine, Ahmadu Bello University Teaching Hospital, Zaria

All correspondence

DrDaboer J.C.

Department of Community Medicine, Jos University Teaching Hospital, P.M.B 2076 Jos, Plateau State

Email:jonathandabor@yahoo.co.uk

Phone:07038959300

Key words: Malaria; eradication; vaccine; review

\begin{abstract}
Summary
Malaria infection has gone through several phases of control and eradication with billions of dollars spent on these efforts. The initial programme of global eradication of the disease was later abandoned by the World Health Organization for technical, economic and political reasons. The disease has thus remained a serious public health problem throughout the developing world. In these areas, conventional methods of control and treatment are becoming ineffective. This is due to the development of resistance of the parasite to conventional drugs and the mosquito vector to the commonly available insecticides.

Recent advances in the fields of biology, monoclonal antibodies and gene cloning, have brought malaria vaccines to the brink of reality. A large amount of data has accumulated concerning parasite biology, hostparasite interactions, immunity and escape mechanisms, and immune responses. Nevertheless, so far this knowledge has not been enough to make us understand how to properly manipulate the whole system to build an effective vaccine. As a result of this, for more than 30 years after the first report of successful vaccination against malaria using radiation-attenuated sporozoites, an effective malaria vaccine is not yet available. However, field and experimental data indicate that it can be developed. This promises to provide the key to the malaria control and eradication obstacle.
\end{abstract}

In this article, we describe the history of malaria control and eradication efforts, the current control strategies and conclude that the development of an effective human vaccine against malaria will provide a leap forward in eradicating the disease.

\section{Introduction}

Malaria is caused by a protozoan parasite of the genus plasmodium. There are four species that are commonly transmitted to man namely plasmodium falciparum, plasmodium vivax, plasmodium ovale and plasmodium malariae but plasmodium falciparum is the most widespread and causes the highest morbidity and mortality. ${ }^{(1)}$ For this reason most control efforts against malaria have been directed against this species. ${ }^{(2)}$ The parasite is transmitted to man through the bite of a female anopheles mosquito. It is a major public health problem in sub-Saharan Africa, Latin America and Asia causing between 300 and 500 million episodes of fever and over I million deaths each year especially among children. ${ }^{(3,4)}$ In addition to its direct effects, it also acts in synergy with and potentiates the effects of malnutrition and other infections especially in tropical African children. At the heart of individuals' vulnerability to malaria is poverty. The combined effect of these is to slow down the physical growth of the affected children, impair their cognitive performance and limit a nation's growth potential. It is estimated that malaria is responsible for slowing down economic growth in Africa by a factor of $1.3 \% .{ }^{(5)}$ Malaria was a major public health problem in most parts of the world including the temperate regions until the mid $20^{\text {th }}$ century when it was eliminated in Europe and North America. $^{(1)}$

Since the identification of the plasmodium species by Laveran in 1880 as the causative agent of malaria fever and the discovery of its oocysts in the gut of the female anopheles mosquito by Ronald Ross in 1897, researchers have never relented in seeking a solution to the malaria burden. ${ }^{(2)}$ For over eleven 
decades mankind has been oscillating between the excitements that accompanied the discovery of new tools to fight the disease and the frustration of failures of these tools to effectively combat malaria. Control efforts have been directed at the mosquito vector, the disease parasite and the human host. ${ }^{(1,6)}$

\section{History of Malaria Control Efforts}

The discovery that mosquito was responsible for the transmission of the malaria parasite meant that if the mosquito could be eliminated malaria transmission would be interrupted. Efforts were therefore made to eliminate mosquito breeding sites from areas around human dwellings. Where this was difficult or impossible attempts were made to prevent man-mosquito contact that might result in mosquito bites. This was done by putting hordes of domestic animals in same sleeping rooms with humans so as to divert the attention of ravaging mosquitoes from biting man. Swamps and marshes were drained and nets were used in homes to prevent man-mosquito contact. ${ }^{(7)}$ With the discovery of paris green and pyrethrum, new armamentaria were added to the arsenals against the mosquito vector. The anti mosquito war became an integrated approach through the use of swamp and marsh drainage, bush cutting, oiling and larviciding (using a larvicidal mixture of carbolic acid, resin and caustic soda). The discovery of quinine and pyrethroids added impetus to the fight against the mosquito. ${ }^{(8,9)}$ Until 1914 when dichlorodiphenyltrichloroethane (DDT) was rediscovered as a new weapon in malaria control, only quinine and the insecticides pyrethrum and paris green were available for the malaria control efforts. By the mid 1950's the debate centered around the best method of malaria control: between tighter netting as canvassed by Battista Grassi, quinine prophylaxis as suggested by Robert Koch or better housing promoted by SP James. ${ }^{(7)}$ Better housing was seen as an indicator of improved socio-economic status. Better housing and improved environmental sanitation would naturally minimize man-mosquito contact that will result in bites. Improvement in nutrition would boost immunity against the parasite. These have been at the root of the successful control of most communicable diseases in the developed countries of the world. Ronal Ross however emphasized vector control as the mainstay of malaria control. ${ }^{(7)}$

Up to 1950 malaria control programmes centered on case management with quinine, personal protection using bed nets and larviciding. Encouraged by the successes of malaria control especially in Europe and the Americas the WHO took over malaria control programme in 1955 by launching an ambitious Global Malaria Eradication programme (GMEP) based on vector control using DDT and prophylaxis using chloroquine. ${ }^{(7,9)}$ This campaign was pursued with vigour especially in Europe and the Americas. An assessment in 1965 showed successes in America and Europe but only partial success was recorded in parts of Asia particularly India and Sri Lanka. It is important to emphasize that these were areas of low malaria transmission. In the countries of Africa where transmission was intense the eradication campaign was considered a failure. This was attributed to loss of political will on the parts of the WHO and the states concerned and the development of resistance of the mosquito vector to the insecticide DDT as well as the plasmodium parasite to chloroquine. In any case no serious large scale eradication efforts were made on the continent of Africa. Even though small scale pilot projects were implemented to test the technical feasibility of the eradication campaign and even though these pilots showed great potential for eliminating malaria, it was concluded that malaria cannot be eradicated from Africa. Additional efforts by the WHO at global malaria eradication were rather unrewarding and by 1969 the WHO was forced to abandon the GMEP. ${ }^{(1)}$

Since the GMEP was abandoned there has been a rise in there burden of the disease globally. Even sulfadoxine-pyrimethamine that replaced chloroquine as first line drug also experienced resistance. ${ }^{(9)}$ The resurgence of malaria was so dramatic that it was also associated with pockets of epidemics in some parts of the world. These included the malaria epidemic which occurred in Sri Lanka between 1968 and 1969 and that which occurred in Madagascar between 1987 and 1988. ${ }^{(1)}$ It was not until 1998 when another major attempt was made in the war against malaria. In this year the Roll Back Malaria (RBM) programme was launched with emphasis on multipronged prevention strategies and prompt case diagnosis and treatment. The new approach lays emphasis on evidence base from research. ${ }^{(10)}$ It can be seen that in about 100 years from when the link between plasmodium species and the mosquito was established, the fight against malaria went round full circle from control to eradication and back to control. 


\section{Current Malaria Control Programme}

The current malaria control programme is based on the RBM which was initiated by African heads of government in April 1995 and later adopted by the WHO at the progamme launch in $1998 .^{(10)}$ The six elements of the RBM include:

1. Evidence-based decisions using surveillance, appropriate response and building community awareness

2. Rapid diagnosis and treatment supporting home care, direct access to effective medicines, and wide availability of health services

3. Multiple prevention using insecticide-treated nets, environmental management to control mosquitoes, and intermittent treatment for making pregnancy safer

4. Focused research to develop new medicines, vaccines and insecticides

5. Well-coordinated action for strengthening existing health services and providing technical support

6. Harmonized action to build a dynamic global movement

The mainstay of the current control programme has been the creation of community awareness, making artemisinin combination drugs available and accessible to those most in need, the use of Long Lasting Insecticide Treated bed Nets (LLINs) especially for children and pregnant women, and research into the development of vaccines. Even though the current strategies of RBM holds a lot of promise there are serious challenges to overcome. The funding opportunities provided by the Global Fund for HIV/AIDS, Tuberculosis and Malaria (GFATM) and Bill \& Melinda Gates Foundation among many other organizations are expected to boost these service and research initiatives.

One of the Challenges being faced is the development of resistance by the mosquito vector against a number of the commonly used insecticides. This means that such insecticides can no longer be effective in combating the vector. The worsening environmental conditions will lead to the proliferation of the mosquito vector and if there is development of substantial resistance to the insecticides in current use malaria control will remain a mirage. ${ }^{(11,12)}$ The challenge is to fund research on a continuous basis so that newer and more effective insecticides will continue to be available.
The second major obstacle is the resistance of the malaria parasite to the commonly available drugs. Quinine is still fairly effective and is currently used in the treatment of severe malaria. However its side effects have limited its common use. Chloroquine which replaced quinine several years ago as the drug of choice has largely been abandoned due to severe resistance of the parasite to the drug. ${ }^{(1,7)}$ The resistance which was first reported in Thailand and Cambodia eventually spread to almost all parts of the world where malaria transmission occurs. Curiously malaria parasite resistance is also being reported for the artemisinin combination drugs in the form of delayed parasite clearance in the western part of Cambodia adjoining Thailand. (13) This is a curious occurrence and research is needed to explain why resistances to the antimalarials usually develop from this axis before spreading to the rest of the world.

The third factor is human behavior. Malaria transmission is favoured in rural areas and urban slums where environmental sanitation is poor. The sanitation situation is even worse in refugee camps and other crowded settlements. ${ }^{(20)}$ Also when human beings clear forests for construction of roads, housing settlements, industries and other purposes they are exposed to large mosquito populations which also leads to increases in malaria transmission. In addition symptom perception and treatment seeking behavior also influence the outcome of malaria in infected individuals. Unfortunately human behavior is difficult to change because it is complex and is influenced by several factors. ${ }^{(14)}$

The implication of the foregoing threats is that the malaria war is far from being won. In the 1990s there was an upward surge in malaria transmission occasioned by the peaking of insecticide and drug resistance and well as deterioration in environmental conditions. In the last decade however, there has been a decline in the prevalence of the disease globally. Several factors may be responsible for this including increased use of ITNs and the use of artemisinin combination therapy. ${ }^{(1)}$ If the resources currently being invested in the control programme is scaled up, there may be a further decline in the disease prevalence. If however the resources decline as happens often, there may be a resurgence of the disease. The only measure that promises to circumvent the issues around insecticide and drug resistance as well as minimize the effect of human behaviour in the control of malaria is the development of an effective vaccine against the malaria parasite. 


\section{Vaccines in Public Health}

Vaccines are often the most cost-effective tools for public health. They have historically contributed to a reduction in the spread and burden of infectious diseases and have played the major part in previous elimination campaigns for smallpox and the ongoing polio, neonatal tetanus and measles initiatives. ${ }^{(15)}$ Yet no effective vaccine for malaria has so far been developed. ${ }^{(16)}$ Despite this, researchers remain hopeful that a preventive or therapeutic malaria vaccine could become available for use in the near future. Optimism is premised on the observation that individuals who are exposed to the parasite in endemic countries develop acquired immunity against disease and death. Such immunity does not however prevent malaria infection; immune individuals often harbour asymptomatic parasites in their blood. Additionally, research shows that if immunoglobulin is taken from immune adults, purified and then given to individuals that have no protective immunity, some protection can be gained. ${ }^{(17)}$ In addition to this, clinical and animal studies have shown that experimental vaccination has some degree of success when using attenuated sporozites and using the RTS, S/AS01 malaria vaccine candidate. ${ }^{(1,17)}$

\section{Potential Targets of a Vaccine}

By their very nature, parasites are more complex organisms than bacteria and viruses, with more complicated structures. The life cycle of the malaria parasite is much more complex than those of bacteria and viruses, presenting initial challenges for the development of an effective vaccine but this also increases the number of potential target sites for a vaccine. Despite the huge number of vaccines available at the current time, there are none that target parasitic infections. ${ }^{(15)}$ The distinct developmental stages involved in the life cycle present numerous opportunities for targeting antigens, thus potentially eliciting an immune response. Theoretically, each developmental stage could have a vaccine developed specifically to target the parasite. The first stage in the parasite life cycle, following inoculation, is a relatively short pre-erythrocytic or hepatic phase. A vaccine at this stage must be able to protect against sporozoite invasion. It should also be able to prevent the development of parasites in the hepatocytes by inducing cytotoxic T-lymphocytes that can destroy the infected liver cells. ${ }^{(17)}$ However, if any sporozoites evade the immune system they will then have the potential to be symptomatic and cause the clinical disease.
The second phase of the life cycle is the erythrocytic or blood phase where merozoites from liver cells are released into the blood stream. The target of a vaccine at this stage is to prevent merozoites from multiplication and/or invasion of red blood cells. Another approach would be to attempt to block the process of erythrocyte adherence to blood vessel walls. It is believed that this process is accountable for much of the clinical syndrome associated with malarial infection; therefore a vaccine given during this stage would be therapeutic and hence administered during clinical episodes to prevent further deterioration. ${ }^{(1)}$

The last phase of the life cycle that has the potential to be targeted by a vaccine is the sexual stage. This would not give any protective benefits to the individual inoculated but would prevent further transmission of the parasite by preventing the gametocytes from producing multiple sporozoites in the gut wall of the mosquito. ${ }^{(18)}$ It therefore would be used as part of a policy directed at eliminating the parasite from areas of low prevalence or to prevent the development and spread of vaccine-resistant parasites. This type of transmission-blocking vaccine is potentially very important. The evolution of resistance in the malaria parasite occurs very quickly, potentially making any vaccine redundant within a few generations. This approach to the prevention of spread is therefore essential. ${ }^{(17,18)}$

\section{Vaccine Development Strategies for the Future}

The development of a vaccine of therapeutic and protective benefit against the malaria parasite requires a novel approach .As to date there are no vaccines available that effectively target a parasitic infection. ${ }^{(16)}$ The focus so far has been predominantly on the use of sub-unit vaccines. The use of live, inactivated or attenuated whole parasites is not feasible and therefore antigenic particles, or subunits, from the parasite are isolated and tested for their ability to elicit an immune response. The majorities of subunits tested are frequently combined with adjuvant and specialized delivery systems to increase the level of immune response. ${ }^{(1)}$ The most recent advances in the field of sub-unit vaccine development include the use of DNA vaccination. This approach involves removing sections of DNA from the parasitic genome and inserting the sequences into a vector. When inoculated the plasmid or attenuated virus is endocytosed into a host cell, the DNA sequence is then incorporated into the host DNA and replicated by protein synthesis. The proteins then produced are expressed on the cell surface membrane of the 
'infected' cell. These bind to the HLA molecules, priming $\mathrm{T}$ cells and therefore creating a population of memory $T$ cells specific to the inoculated DNA sub-unit. This technique has been shown to produce a high rate of $\mathrm{T}$ cell response but poor level of antibody production. The efficacy of DNA vaccines can be assessed using an ELISPOT assay. ${ }^{(18)}$ The development of this method of testing for immune responses is extremely beneficial when examining the potential efficacy of a vaccine candidate and is hoped to enable critical analysis of the mechanisms that provide 'partial' protection, thus facilitating a greater understanding of vaccine technology. This approach of potentially allowing the modification of vaccine candidates to improve development techniques and further scientific understanding is known as 'iterative development'. The advantage of DNA vaccines over classical attenuated vaccines are numerous and include being able to mimic MHC class $1 \mathrm{CD} 8+\mathrm{T}$ cell specific responses that potentially could reduce some of the safety concerns associated with vaccine therapy and additionally provide a substantial reduction in production cost and due to the nature of DNA vaccines, increased ease of storage. ${ }^{(1,18)}$

The most successful candidate developed to date is the RTS,S recombinant vaccine. The RTS,S/AS02A, one of the key vaccines produced using this technique, has been used in field trials in The Gambia. ${ }^{(19)}$ Three repeat doses were administered in the 6 months leading up to the period of highest malaria transmission. ${ }^{(20)}$ The vaccine efficacy was reported at approximately $71 \%$ during the first 2 months of follow-up, but falling to $0 \%$ in the last 6 weeks in 250 male volunteers. ${ }^{(1,21)}$

\section{Conclusion}

The malaria scourge is as old as mankind. In spite of the myriad of tools that have been developed against it, the disease remains a major public health problem especially among the poor populations of sub-Saharan Africa and Asia. Conventional tools against the disease have proved ineffective in eradicating or even controlling the disease. However preliminary results from research and field trials suggest that a preventive vaccine against malaria is in sight. When available for public use this will provide the answer to the malaria eradication question that has occupied the mind of researchers and clinicians for decades.

\section{References}

1. Greenwood BM, Fidock DA, Kyle DE, Kappe SHI, Alonso PL, Collins FH et al. Malaria: progress, Perils and Prospects for Eradication. J Clin Invest 2008; 118(4):1266-1276

2. Centers for Disease Control and Prevention. History of Malaria Control. Available from http://www.cdc.gov.malariainfo Last accessed $22 / 05 / 2010$

3. United Nations. Coming to Grips with Malaria in the New Millenium. UN Millenium Project 2005. Task Force on HIV/AIDS, Malaria, TB and Access to Essential Medicines. Working Group on Malaria

4. World Health Organization. World Malaria Report 2005. Geneva. RBM/WHO/UNICEF 2005.

5. United Nations. Global Burden of Malaria. MilleniumProject. Available from http://www.unmilleniumproject.orgLast accessed 22/05/2010

6. Progress in Malaria Control. Global Malaria Action P l a n. Ava i la ble from http://www.rollbackmalaria.org/gmap/1-3.html Last accessed 16/05/2010

7. History of Malaria. Available from http://www.malariasite.com/malaria/history.htm Last accessed 28/05/2010

8. Centres for Disease Control and Prevention. Malaria. World Malaria Report 2009. Available from http://www.cdc.gov/malaria/

9. Matthys B, Sherkanov T, Karimov SS, Khabirov Z, Mostowlansky T, UTzinger J et al. History of Malaria in Tajikistan and Rapid Malaria Appraisal in an Agroecological setting. Malaria Journal 2008; 7: 217- 224

10. UNICEF. The Roll Back Malaria Partnership. Available from http://www.unicef.org

11. Knipling EF. Present Status of Mosquito Resistance to Insecticides. Am J Trop Med Hyg 1952; 1(3): 389-394

12. Wikipedia. Pesticide Resistance. Available from http://wikipedia.org

13. World Health Organization. Drug Resistance could set back malaria control success. WHO News Release 2009. Available from http://www.who.int

14. Mwenesi HA. Sociocultural and Behaviuoral Issues in the Treatment and Prevention of Malaria. WHO/TDR 2008

15. World Health Organization. Immunization Against Diseases of Public Health Importance. Fact sheet No 288, 2005

16. Young JF, Poste G. The Prospects for a Human Malaria Vaccine. Trends in Biotechnology 1988; 6(3): 63-68

17. Chauhan VS Vaccines for Malaria-Prospects and Promise. Current Science 2007; 92(11):1525-1534 
18. Breman JG Plowe C. A malaria vaccine for control: more progress. The Journal of Infectious Diseases 2009; 200(3):317-320

19. Ndugwa RP, Ramroth H, Muller O, Jasseh M, Sie A, Kouyate B et al. Comparism of allcause and malaria specific mortality from two West African countries with different malaria transmission patterns. Malaria Journal 2005; A v a i $1 \mathrm{a} \mathrm{ble}$ f $\mathrm{r}$ o m http://www.malariajournal.com/content/7/1/ 15 Last accessed 01/06/2010
20. Rowland M, Nosten F. Malaria epidemiology and control in refugee camps and complex emergencies. Annals of Tropical Medicine and Parasitology 2001; 95 (8):741-754

21. Omar SA, Adagu IS, Gump DW, Ndaru NP, Warhurst DC. Plasmodium falciparum in Kenya: high prevalence of drug resistanceassociated polymorphisms in hospital admissions with severe malaria in an epidemic area. Annals of Tropical Medicine and Parasitology 2001; 95(7):661-669 